\title{
Empirical validation of a queueing approach to uninterrupted traffic flows
}

\author{
Tom Van Woensel ${ }^{1}$ and Nico Vandaele ${ }^{2}$ \\ 1 Eindhoven University of Technology, Den Dolech 2, Pav. F05, Eindhoven NL 5600 MB, \\ The Netherlands, (e-mail: t.v.woensel@tm.tue.nl) \\ 2 University of Antwerp, Prinsstraat 13, 2000 Antwerpen, Belgium
}

Received: April 2005 / Revised version: June 2005

\begin{abstract}
In this paper, the use of queueing theory for modeling uninterrupted traffic flows is evaluated. Empirical data on speeds and flows are used to evaluate speeds generated by the different queueing models. Using the Theil inequality coefficient as evaluation criterion, the speeds generated by the queueing models are compared to the empirical speeds. Queueing models that best fit the observed speeds are obtained. It appears that traffic flow on a highway during non-congested hours is best described using a $M / G / 1$ queueing model. During the congested hours however, the state dependent queueing $G I / G / z$ models are more realistic. Because the queueing models describe the empirical data well, they can also be used to evaluate potential improvements in existing traffic conditions.
\end{abstract}

Key words: Traffic flows, queueing theory, validation

MSC classification: $60 \mathrm{~K} 30,68 \mathrm{M} 20$

\section{Introduction}

The combination of increasing traffic (demand) on the existing road networks together with a stagnation of investments in new infrastructures (supply), leads to an inevitable increase of congestion. This in turn leads to an increase in travel time, decreasing vehicle flows, higher fuel consumption, negative environmental effects, etc. In general, three strategies for this demand-supply gap are possible (Button 1993). 
First, the existing road networks (supply) can be expanded. Investments in new roads lead to an increase in capacity, resulting in a (temporary) decrease of congestion. New investments can be useful to dispose of existing bottlenecks, but are not a structural solution to congestion because increased supply will probably result in a more than proportional increase of demand. Secondly, the number of cars on the roads (demand) can be decreased. This can for example be achieved by developing pricing tools (congestion pricing), facilitating public transport, etc. This approach is a more structural solution to congestion than the first one. It is, however, in practice, very difficult to decrease traffic demand or to force a shift to public transport. A third solution is to efficiently manage the existing traffic flows on the existing road network i.e. traffic management. Efficiently tuning demand and supply will lead to a better use of the capacity and a better control of traffic demand. This third solution can for example be achieved by a temporary and local intervention on supply and demand. Measures that can be implemented in this solution approach are arrival management, DRIPS (Dynamic Route Information Panel Systems) that give information about the state of the road network, incident management (quick response to accidents), dynamic adjustment of the number of lanes, time-dependent pricing policies, etc. Of course the global solution for congestion should be a mixture of the above partial solutions.

Quantifying the above actions that cope with congestion is not straightforward however. The first step should always be a good understanding of the complex phenomenon of congestion itself (e.g. what are its driving forces?). In this paper, traffic congestion is modeled using queueing models. This analytical basis (rather than an empirical one) has the advantage to adequately pinpoint possible problems and allowing to perform sensitivity analysis, what-if questions, etc. Once traffic is analytically analyzed and modeled, the second step tries to adequately improve the traffic conditions, using these mathematical models (e.g. what is the effect of certain actions?). Starting from the existing traffic state, the potential improvements can be quantified and compared (and implemented).

Traffic flow modeling is divided into two primary types of flows: uninterrupted versus interrupted traffic flows (Transportation Research Board 1996). The first type, uninterrupted flows, is defined as all the flows regulated by vehicle-vehicle interactions and interactions between vehicles and the roadway. For example, vehicles traveling on a highway are participating in uninterrupted flows. Interrupted flows, the second type of traffic flows, are all flows regulated by an external means, such as a traffic signal. Under interrupted flow conditions, vehicle-vehicle interactions and vehicle-roadway interactions play a secondary role in defining the traffic flow. Understanding what type of flow is occurring in a given situation will lead to different methods for analyzing traffic situations. In this paper, only uninterrupted flows are considered, i.e. only traffic on highways is considered and modeled. Traditionally, uninterrupted traffic flows are modeled empirically: speed and flow data are collected for a specific road and econometrically fitted into curves (Daganzo 1997). This traditional approach is limited in terms of predictive power and sen- 
sitivity analysis. Queueing theory is almost exclusively used to describe traffic behavior at signalized and unsignalized intersections (Heidemann 1991, 1994 and 1997). However, Vandaele et al. (2000) and Heidemann (1997), showed that queueing models can also be used to explain uninterrupted traffic flows and thus offering a more practical approach, useful for sensitivity analysis, forecasts, etc. Jain and Smith (1997) describe in their paper a state-dependent $M / G / C / C$ queueing model for traffic flows. Also a lot of research is done on a travel time-flow model originating from Davidson (1978), which is also based on some concepts of queueing theory (see also Akçelik 1991 and 1996).

In this paper, the applicability of the queueing models for traffic flows is evaluated. Empirical data for the speeds, flows and densities are used to evaluate the speeds generated by the different queueing models. In Sect. 2, the general methodology for modeling traffic flows with queueing models is explained in detail. The third section describes the chosen evaluation criteria. In Sect. 4, the datasets used and the cleaning operations performed are described. In Sect. 5, results are reported in two steps: first, the results for the calibration phase are presented and secondly, the results of the validation phase are discussed. This paper ends with some conclusions.

\section{Queueing models for traffic flows}

It is often observed that the speed for a certain time period tends to be reproduced whenever the same flow is observed. Based on this observation, it seems reasonable to postulate that, if traffic conditions on a given road are stationary, there should be a relationship between flow, speed, and density. This relationship results in the concept of speed-flow-density diagrams. These diagrams describe the interdependence of traffic flow $(q)$, density $(k)$ and speed $(v)$. The seminal work on speedflow-density diagrams was the paper by Greenshields in 1935. Using well-known formulas of queueing models, these diagrams can be constructed (Fig. 1).

The speed-flow diagram is the envelope of all possible combinations of the effective speed and traffic flow. Figure 1 (upper right) illustrates that, although every speed $v$ corresponds with one traffic flow $q$, the reverse is not true. There are two speeds for every traffic flow: an upper branch $\left(v_{2}\right)$ where speed decreases as flow increases and a lower branch $\left(v_{1}\right)$ where speed increases. Intuitively it is clear that, as the flow moves from 0 (at free flow speed $v_{f}$ ) to $q_{\text {max }}$, congestion increases but the flow rises because the decline in speed is over-compensated by the higher traffic density. If traffic tends to grow past $q_{\max }$, flow falls again because the decline in speed more than offsets the additional vehicle numbers, further increasing congestion (Daganzo 1997). The flow-density diagram and the speed-density diagrams are an equivalent representation and can be interpreted in the same way.

In a queueing approach to traffic flow analysis, roads are subdivided into segments, with length equal to the minimal space needed by one vehicle on that road (Fig. 2). Define $k_{j}$ as the maximum traffic density (i.e. maximum number of cars on 


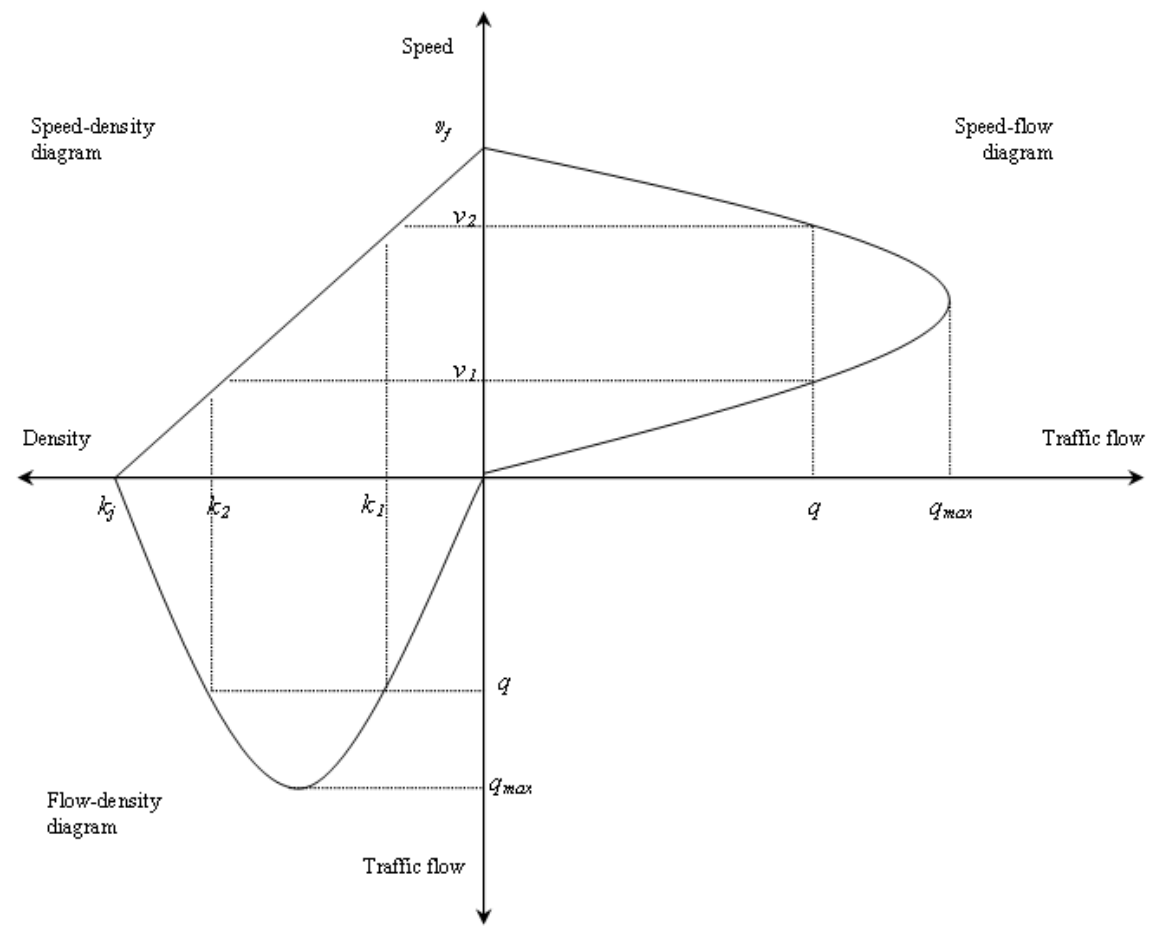

Fig. 1. The relations between the speed-flow, the speed-density, and the flow-density diagrams

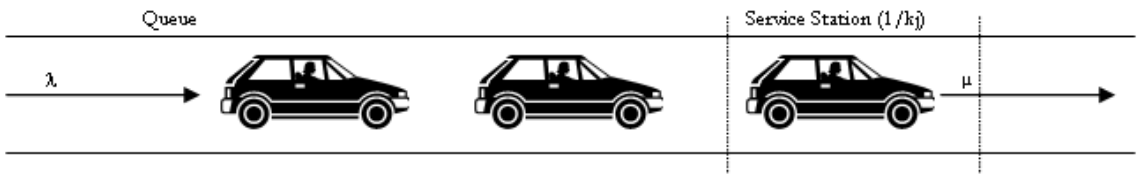

Fig. 2. Queueing representation of traffic flows

a road segment). This segment length is then equal to $1 / k_{j}$ and matches the minimal space needed by one vehicle on that road. Each road segment is then considered as a service station, in which vehicles arrive at a certain rate $\lambda$ and get served at another rate $\mu$ (Vandaele et al. 2000; Van Woensel et al. 2001; Heidemann 1997).

Vandaele et al. (2000) developed different queueing models. In this paper, queueing models are referred to using the Kendall notation, consisting of several symbols, e.g. $G I / G / z$. The first symbol is shorthand for the distribution of inter-arrival times, the second for the distribution of service times and the last one indicates the number of servers in the system. The $M / M / 1$ queueing model (exponential arrival and service rates) is considered as a base case, but due to its specific assumptions regarding the arrival and service processes, it is not useful to describe real-life situations. Relaxing the specifications for the service process 
of the $M / M / 1$ queueing model, leads to the $M / G / 1$ queueing model (generally distributed service rates). Relaxing both assumptions for the arrival and service processes results in the $G I / G / z$ queueing model (with $z$ being the number of servers). Moreover, following Jain and Smith (1997), a state dependent $G I / G / z$ queueing model is considered. This model assumes that the service rate is a (linear, exponential, etc.) function of the traffic flow. In this case vehicles are served at a certain rate, which depends upon the number of vehicles already on the road.

Following Heidemann (1997), the arrival rate $\lambda$ is defined as the product of the traffic density $k$ and the free flow speed $v_{f}$, or $\lambda=k v_{f}$. Similarly, the service rate $\mu$ is defined as the product of free flow speed $v_{f}$ with the maximum traffic density $k_{j}$, or $\mu=k_{j} v_{f}$. Vandaele et al. (2000) and Heidemann (1997) showed that the speed $v$ can be calculated by dividing the length of the road segment $\left(\frac{1}{k_{j}}\right)$ by the total time in the system $(W)$ :

$$
v=\frac{1 / k_{j}}{W}
$$

The total time in the system $W$ in equation (1) is different depending upon the specific queueing model used. The total time spent in the system $W$ equals the sum of the waiting time $W_{q}$ and the service time $W_{p}$. For the $G I / G / z$ queueing models, no exact formulas for $W_{q}$ are available and one must rely on approximations. Here, three approximations are considered: the Kraemer-Lagenbach-Belz $(K L B)$ approximation (1976), the heavy traffic or Kingman approximation $(K)(1964)$ and the Whitt $(W)$ approximations (1993) are used.

In general, Eq. (1) can be rewritten in the following basic form (see Van Woensel 2003 for the details):

$$
v=\frac{v_{f}}{1+\Omega}
$$

Equation (2) shows that the speed is only equal to the free flow speed $v_{f}$ if the factor $\Omega$ is zero. For positive values of $\Omega, v_{f}$ is divided by a number strictly larger than 1 and speed is reduced. The factor $\Omega$ is thus the influence of congestion on speed. High congestion (reflected in a high $\Omega$ ) leads to lower speeds than the maximum. The factor $\Omega$ is a function of a number of parameters depending upon the queueing model chosen: the traffic intensity $\rho$, the coefficient of variation of service times $c_{s}$, the coefficient of variation of inter-arrival times $c_{a}$, the jam density $k_{j}$ and the free flow speed $v_{f}$. High coefficients of variation or a high traffic intensity will lead to a value of $\Omega$ strictly larger than zero. Actions to increase speed (or decrease travel time) should then be focused on decreasing variability or on influencing traffic intensity, for example by manipulating the arrivals (arrival management and ramp metering). Table 1 shows the specific form of $\Omega$ for each queueing model. Instead of using a fixed service rate $\mu$ for the queueing models, the service rate can also be made a function of the traffic flow $q$. This results in the state dependent queueing models, with the state being the number of vehicles $q$ on the road at that moment. 
Table 1. The specific form of $\Omega$ for each queueing model

\begin{tabular}{ll}
\hline Queueing model & $\Omega$ \\
\hline$G I / G / 1 K L B$ & $\frac{\rho}{(1-\rho)} \frac{\left(c_{a}^{2}+c_{s}^{2}\right)}{2} \exp \left[\frac{-2(1-\rho)\left(1-c_{a}^{2}\right)^{2}}{3 \rho\left(c_{a}^{2}+c_{s}^{2}\right)}\right]$ \\
\hline$G I / G / z K$ & $\left(\frac{c_{a}^{2}+c_{s}^{2}}{2}\right)\left(\frac{\rho(\sqrt{2(z+1)}-1)}{z(1-\rho)}\right)$ \\
\hline$G I / G / z W$ & $\phi\left(\frac{c_{a}^{2}+c_{s}^{2}}{2}\right) W_{q M / M / z}$ \\
& $\begin{array}{l}\text { With } \phi \text { a correction factor defined in Whitt (1993) } \\
\text { and } W \text { qM } / M / z \text { the formula for the waiting time in an } \\
\end{array}$ \\
& $M / M / z$ queue (See Vandaele et al. 2000). \\
\hline
\end{tabular}

For a detailed discussion of the queueing models and some applications, the interested reader is referred to Vandaele et al. (2000), Van Woensel (2003) and Van Woensel et al. (2001).

\section{Evaluation criteria}

The reliability of the queueing models depends upon the ability to produce results close to reality. The process of determining whether the queueing models generate speeds close enough to real speeds is achieved through the validation of the models. This is an iterative process involving calibration of the model parameters, comparing the output with the real speeds and improving the model until the accuracy is acceptable. When several days of observations are available, the calibration and the final validation of the queueing models are done on different datasets. This will generate the trust that the model is not only accurate for the day(s) for which it was calibrated but that the calibration also holds in general. To validate the queueing model, it should be able to emulate the actual traffic process and produce a series of speeds which are close to the actual observed speeds. In short, to evaluate the speed difference between the speeds obtained from the queueing models and the ones empirically observed, an evaluation tool is needed. Some of the most used tool in traffic simulation is the root mean square error, the root mean square percentage error, residual variance, etc. These statistical methods and techniques for validating models are presented in most textbooks and specialized papers (see e.g., Kleijnen 1995; Balci 1998; Law and Kelton 2000). These tools however have a drawback because they square the errors and as a consequence have the risk of overemphasizing large errors (Barceló 2001). Alternatively, the correlation coefficient cannot be used to evaluate either because it is inadequate to evaluate the difference between the queueing speed and the observed speed. For example, the two series of speeds can be perfectly correlated but have a (large) constant difference.

In this paper, the Theil inequality coefficient (Theil 1966), which does take into account for this disproportionate weight of large errors is used. Moreover, in 
situations where little data is available it is possible that neither the observed speeds nor the queueing speeds are independent. The Theil coefficient can still be used in these situations. This coefficient is thus a more significant indicator on how similar the observed and simulated series are compared to the other measures mentioned. Moreover it is a measure that can be used adequately to compare and evaluate the similarities of two different time series and it overcomes the major drawbacks of the other tools mentioned (Barceló 2001). The Theil coefficient Theil $l_{v q, v o b s}$ of the queueing speed $v_{q}$ and the observed speed $v_{o b s}$ is defined as:

$$
\text { Theil }{ }_{v q, v o b s}=\frac{\sqrt{\frac{\sum\left(v_{q}-v_{o b s}\right)^{2}}{n}}}{\sqrt{\frac{\sum v_{q}^{2}}{n}}+\sqrt{\frac{\sum v_{o b s}^{2}}{n}}}
$$

This measure is frequently used to assess the accuracy of econometric forecasts compared to actual observations. The forecasts are in our case the speeds calculated with the queueing model and the actual observations are then the observed speeds. If all forecasts are on target, the value of $T h e i l_{v q, v o b s}$ is 0 . If however the forecasts are very inaccurate, the Theil coefficient goes to 1 (Holden et al. 1990). Concluding, a low value of $T h e i l_{v q, v o b s}$ means that the speeds obtained from the queueing models are close to the observed speeds.

One extra advantage of using the Theil inequality coefficient is the decomposition into the three proportions of inequality. These proportions are useful as a means of breaking down the error down into three different characteristic sources: bias (error due to the difference in means), variance (error due the difference in variance) and covariance (error due to randomness) (Holden et al. 1990). The bias proportion gives information about how far the mean of the series of queueing speeds $v_{q}$ is from the mean of the actual series of observed speeds $v_{o b s}$. The variance proportion gives information about how far the variation of the series of queueing speeds $v_{q}$ is from the mean of the variation of the series of observed speeds $v_{o b s}$. The covariance proportion measures the remaining unsystematic errors. The bias, variance and covariance proportions all add up to one. The best results are those for which the bias and variance proportions are small or where the covariance proportion is the largest.

\section{The datasets}

A dataset collected by the ministry of transportation of the Flemish Government (Belgium) is used in the analysis. This ministry is responsible for collecting and reporting the counting data results on all Flemish highways. The original dataset contains minute-per-minute observations for the period from February 7, 2000 to February 13, 2000. For each minute, data are available for all counting points on the Beltway $R 1$ around Antwerp and all counting points immediately before the Beltway $R 1$ that are located on highways leading to the Beltway $R 1$. More 
Table 2. The decomposition of the Theil coefficient

\begin{tabular}{|c|c|}
\hline Proportion & \\
\hline Bias & Theil ${ }_{\text {bias }}=\frac{\left(\overline{v_{q}}-\overline{v_{o b s}}\right)^{2}}{\frac{1}{n} \sum\left(v_{q}-v_{o b s}\right)^{2}}$ \\
\hline Variance & Theil $_{\text {var }}=\frac{\left(\sigma_{q}-\sigma_{\text {sobs }}\right)^{2}}{\frac{1}{n} \sum\left(v_{q}-v_{o b s}\right)^{2}}$ \\
\hline Covariance & Theil $_{\text {cov }}=\frac{2 *(1-r) * \sigma_{q} * \sigma_{\text {sobs }}}{\frac{1}{n} \sum\left(v_{q}-v_{o b s}\right)^{2}}$ \\
\hline & $\begin{array}{ll}\sigma_{q} & \text { The standard error of the queueing speeds } \\
\sigma_{\text {obs }} & \text { The standard error of the observed speeds } \\
r & \text { The correlation coefficient of the two series }\end{array}$ \\
\hline
\end{tabular}

specifically, the dataset contains all data for the number of trucks, the number of passenger cars, the speed and the density. All these data are available for each counting point on a minute-per-minute basis for each direction and for each lane. As a consequence, for each counting point 10080 records (60 minutes times 24 hours times 7 days) are available. The counting points are distributed all over the ring road $R 1$ around Antwerp, starting at the on-and off-ramp complex at the left bank of Antwerp (at highway E17) and going to the end of the ring road $R 1$ where highway $E 19$ starts. Besides data for the ring road $R l$, data is also available for all incoming highways (i.e. from/to Gent on the left bank of Antwerp: E17; from/to Brussels: $A 12$ and $E 19$; from/to Liege: $E 34$; from/to Breda: $E 19)$. In total, 64 counting points were available in the original dataset, resulting in a total dataset size of over 600000 records (64 counting points times 10080 records for each counting point).

As a first step, one counting point of the total dataset (Kaaien-Merksem in the North of Antwerp, in the direction of Antwerp coming from Breda, The Netherlands) is randomly selected. All trucks and passenger cars are converted to a vehicle equivalent, i.e. cars are 1 vehicle equivalent and trucks are 2 vehicle equivalents (see e.g., Daganzo 1997). The concept of the vehicle equivalents is based on observations of freeway conditions in which the presence of heavy vehicles (e.g., trucks, buses, etc.) create less-than-ideal conditions, including longer and more frequent gaps of excessive lengths both in front of and behind these heavy vehicles. Physical space taken up by a heavy vehicle is typically two to three times greater in length than that of a passenger car (Transportation Research Board 1996).

In a second step all minute-per-minute observed flows and speeds are aggregated to a 10-minute time interval and over the different lanes. More specifically, the minute-by-minute speeds are averaged over 10 minutes and the minute-byminute flows are summed over 10 minutes (see e.g., Transportation Research Board (1996)). In the dataset, missing values were denoted with a value -9999 . Missing values are replaced by the mean value of the other observations during the time interval under consideration (i.e., 10 minutes). The speed for the time interval is calculated as the mean of the minute-per-minute speed over all lanes. This pro- 
cedure works well for time intervals with little missing observations. Due to this limitation, not enough observations are available during the hours 1 to 4 and 24 to correct for the missing data.

To increase the usability of the dataset, one day is selected to calibrate the models in the analysis: February 8, 2000. The validation phase is performed with data collected on February 10, 2000 at the same counting point.

\section{Queueing speed versus observed speed}

Using the queueing models described, one can calculate the speeds for the observed flows (from the dataset) and compare them with the real observed speeds. As such for the same observed flow, two speeds are available: an observed speed and a queueing model speed. The difference between the two speeds series over all flow observations is then evaluated using the Theil coefficient. By changing the input parameters, the queueing model that minimizes the Theil coefficient is obtained (Fig. 3). The parameters in the evaluation are the queueing model itself $(M / M 1$, $M / G / 1, G I / G / z$ and the state dependent ones, refered to as $S D)$ and its respective parameters (the traffic intensity $\rho$, the coefficient of variation of service times $c_{s}$, the coefficient of variation of inter-arrival times $c_{a}$, the jam density $k_{j}$ and the free flow speed $v_{f}$ ). When the best model is chosen using this methodology, the Theil coefficient is decomposed into its three proportions to identify the source of the error made.

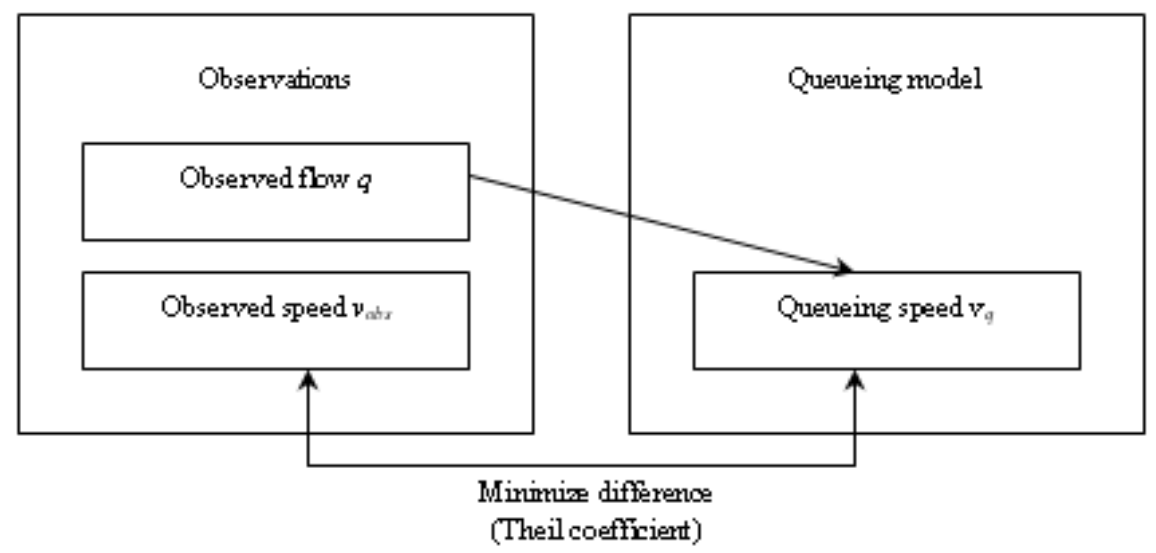

Fig. 3. Evaluation methodology

In a first step, the queueing models are calibrated. Calibration means determining the right values for the parameters governing the queueing models. In a second step, the calibrated queueing models are used to calculate the speeds for a second similar day in the dataset (i.e., validation). 
Table 3. Results for the unstratified dataset (Calibration)

\begin{tabular}{cccc}
\hline Model & Approximation & Theil & Correlation \\
\hline$M / M / 1$ & & 0.08948 & 0.5450 \\
$M / G / 1$ & & 0.08970 & 0.5394 \\
$G I / G / 1$ & $K L B$ & 0.11722 & 0.7348 \\
& $K$ & 0.11003 & 0.7011 \\
& $W$ & 0.10730 & 0.7074 \\
$G I / G / 3$ & $K$ & 0.11003 & 0.7808 \\
& $W$ & 0.11352 & 0.7741 \\
$S D G I / G / 1$ & $K L B$ & 0.11351 & 0.5184 \\
& $K$ & 0.08011 & 0.5253 \\
$S D G I / G / 3$ & $W$ & 0.07974 & 0.5239 \\
& $K$ & 0.08012 & 0.5244 \\
& $W$ & 0.07906 & 0.5185 \\
\hline
\end{tabular}

\subsection{Calibration}

This section describes the results of the calibration phase of the queueing models. As explained above, the observed flow together with the parameters of the chosen queueing model is the basic input needed. All values of the parameters in the models are evaluated within a certain range: $c_{a}$ and $c_{s}$ both ranging between 0.5 and 1 with step-size $0.05, k_{j}$ is varied from $60 \mathrm{veh} / \mathrm{km}$ to $100 \mathrm{veh} / \mathrm{km}$ with a step-size of 1 veh/ $\mathrm{km}$ and $v_{f}$ is varied from $80 \mathrm{~km} / \mathrm{hr}$ to $150 \mathrm{~km} / \mathrm{hr}$ with a step-size of $1 \mathrm{~km} / \mathrm{hr}$. The ranges for these values are partially found in the literature (Transportation Research Board 1996) and are partially based on some feasibility constraints taking into account physical space on the road (e.g., for the jam density), technology of cars (e.g., maximum speeds). Due to the limited number of possible combinations and values of the parameters, all parameter combinations (i.e. a full enumeration) are evaluated and the combination with the lowest Theil coefficient is retained and labelled as best. Moreover, due to the analytical character of the queueing models, the computation time needed to find the best parameter combination for the queueing model was always limited (less than one minute).

For the first day of the described dataset, the queueing speeds for the observed flows are obtained. Each queueing model with its respective parameter combination is evaluated in terms of the Theil coefficient. The best parameter setting that minimizes the Theil coefficient is then retained. Table 3 shows the key results for the different queueing models. In general, the state dependent $G I / G / z$ queueing models $(S D G I / G / z)$ result in speeds closest to the observed speeds (as reflected in the lowest Theil coefficient).

In all results, one common factor can be distinguished: in hours 7 to 9 and 16 to 18 the speeds obtained with the queueing models lie far away from the real observed speeds. These hours are typically congestion hours with large peaks in flow (see also Van Woensel 2003). Especially during these hours the speed from the queueing model was deviating largely from the observed speeds. The reason is that apparently one queueing model with one parameter setting is inadequate to represent the traffic 
Table 4. Results for the stratified dataset (Calibration)

\begin{tabular}{ccccc}
\hline Model & Approximation & No & Morning & Evening \\
\hline & & congestion & congestion & congestion \\
$M / M / 1$ & & 0.03928 & 0.04650 & 0.05939 \\
$M / G / 1$ & & 0.03440 & 0.04552 & 0.08375 \\
$G I / G / 1$ & $K L B$ & 0.09853 & 0.07357 & 0.14962 \\
& $K$ & 0.08401 & 0.06169 & 0.14546 \\
& $W$ & 0.07806 & 0.05683 & 0.14765 \\
$G I / G / 3$ & $K$ & 0.10778 & 0.07830 & 0.12028 \\
& $W$ & 0.09876 & 0.07033 & 0.11551 \\
$S D G I / G / 1$ & $K L B$ & 0.05335 & 0.05070 & 0.03656 \\
& $K$ & 0.05640 & 0.06010 & 0.03634 \\
$S D G I / G / 3$ & $W$ & 0.05564 & 0.06021 & 0.03590 \\
& $K$ & 0.04809 & 0.03516 & 0.03760 \\
& $W$ & 0.05137 & 0.04647 & 0.03641 \\
\hline
\end{tabular}

Table 5. The Theil decomposition (Calibration)

\begin{tabular}{ccccc}
\hline & & \multicolumn{3}{c}{ Decomposition } \\
\hline Total dataset & Theil & Bias & Variance & Covariance \\
$S D G I / G / 3 W$ & 0.07906 & 0.00787 & 0.14581 & $\mathbf{0 . 8 4 6 3 1}$ \\
\hline & \multicolumn{4}{c}{ Decomposition } \\
\hline Stratified dataset & Theil & Bias & Variance & Covariance \\
No congestion & 0.03440 & 0.13453 & 0.11088 & $\mathbf{0 . 7 5 4 5 9}$ \\
Morning congestion & 0.03516 & 0.40983 & 0.01921 & $\mathbf{0 . 5 7 0 9 6}$ \\
Evening congestion & 0.03590 & 0.00557 & 0.13437 & $\mathbf{0 . 8 6 0 0 6}$ \\
Complete day & 0.03472 & 0.02342 & 0.02495 & $\mathbf{0 . 9 5 1 6 3}$ \\
\hline
\end{tabular}

process for the full day. It is often observed that the traffic process during the congested hours is different than in the uncongested hours (Button 1993). To verify for this phenomenon and to increase the model performance, the dataset is split up into three more coherent subsets: i.e. morning congestion, evening congestion and all other hours (no congestion) (see also Ichouia et al. 2003 for a similar approach). Then, for each subset the queueing models are again evaluated in terms of the Theil coefficient. Table 4 summarizes all relevant results for this stratified set.

Combining the best results for each considered period of the day (no congestion, morning congestion and evening congestion), the best fit for the whole day is obtained. More specifically, the best queueing model for the uncongested hours of the day is the $M / G / 1$ queueing model. During the congested hours however, the state dependent queueing models $(S D)$ seem to perform better. The overall Theil coefficient using the above models for the different periods of the day, is 0.03472 . Figure 4 compares the observed speed and the queueing speed. The Theil inequality coefficient is decomposed into the three proportions of inequality: bias, variance and covariance proportion (Table 5).

It appears that $85 \%$ of the error made when using one queueing model for the whole day can be attributed to random errors (covariance proportion). Using the 


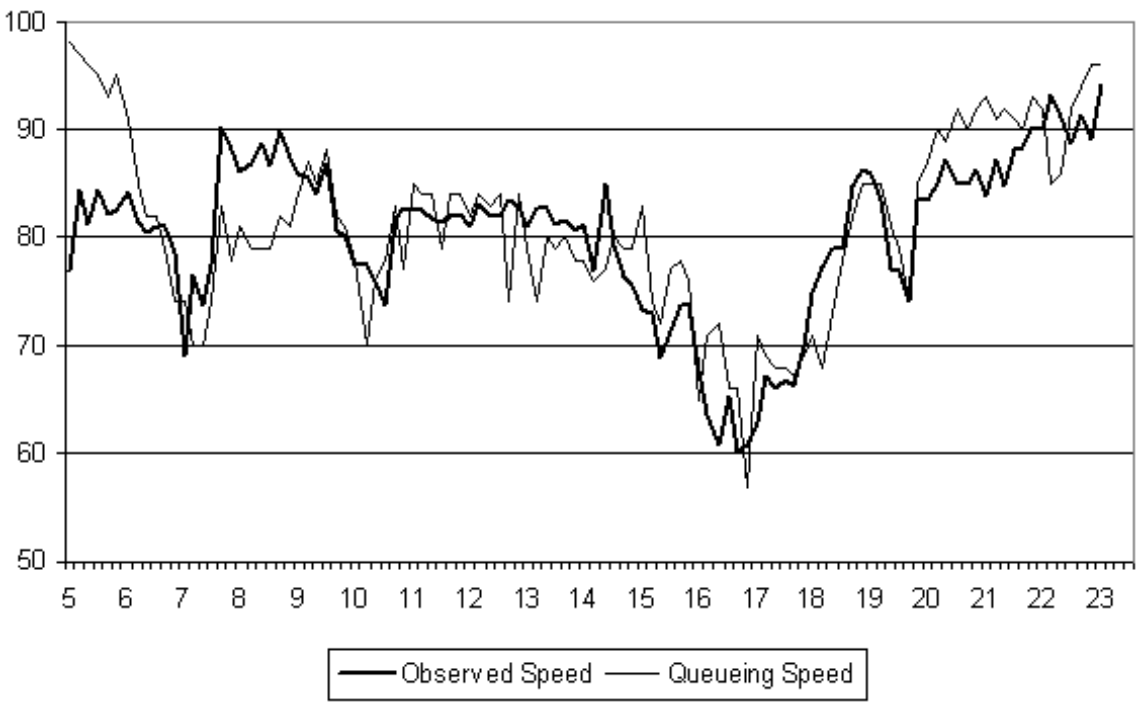

Fig. 4. Queueing speed vs Observed speed (Calibration)

Table 6. Results for the stratified dataset (Validation)

\begin{tabular}{ccccc}
\hline & & \multicolumn{3}{c}{ Decomposition } \\
\hline Total dataset & Theil & Bias & Variance & Covariance \\
$S D G I / G / 3 W$ & 0.09132 & 0.20343 & 0.00000 & 0.79657 \\
\hline & & \multicolumn{3}{c}{ Decomposition } \\
\hline Stratified dataset & Theil & Bias & Variance & Covariance \\
No congestion & 0.04953 & 0.00421 & 0.29180 & 0.70399 \\
Morning congestion & 0.05847 & 0.00829 & 0.18053 & 0.81118 \\
Evening congestion & 0.09553 & 0.59873 & 0.32716 & 0.07411 \\
Complete day & 0.04932 & 0.00657 & 0.00601 & 0.98742 \\
\hline
\end{tabular}

stratified sample and the period specific queueing models (improved model), does not change this results in terms of the decomposition.

\subsection{Validation}

In a second step, the calibrated queueing models obtained above are used to evaluate another comparable day. In other words, the queueing models and its parameters obtained in the calibration step are used again with a different day. Again, the evaluation is done in terms of the Theil coefficient and its decomposition. Additionally, the correlation coefficient is given. Table 6 gives the results and the Theil decomposition of the calibrated queueing models. 
In terms of the Theil inequality coefficient, the queueing models lose a bit of their power to generate speeds that are close to the observed speeds. When looking at the Theil decomposition, only for the evening congestion, it appears that the queueing model used is not adequate to model the speeds on this day. Because the largest part of the error made lies in the bias proportion, it is suspected that the characteristics of the evening congestion are substantially different from the day used for the calibration of the models. This is typically the case if the process during this time period is not stationary. In general, the queueing models seem to be fairly robust if the underlying situation for the different days is not very different. These results are similar to what is found by Van Woensel (2003).

\section{Conclusions}

In this paper, the applicability of queueing models for traffic flows is validated. Using empirical speed and flow data, the best queueing model is searched for such that the difference between the observed speed and the queueing speed is minimal. The mathematical instrument used to measure this difference is the Theil coefficient. It appeared that an overall analysis using one single queueing model for the whole day is inadequate. Therefore the analysis is extended towards a more stratified sample based on the hours of the day, using different queueing models for different periods of the day. The rationale is that for some hours of the day (congestion hours) the best queueing model will be different. Model validation is central in this paper, parameter validation is of second importance here. The parameters are optimized using the Theil coefficient on a limited number of observations. This approach confirms that the use of queueing models is justified to model traffic flows. On the other hand, if one (e.g., policy makers) would use these models then a periodic re-evaluation of the parameters used will be necessary. Then much more data is needed to adequately pinpoint the values of the parameters. Other research via simulation showed that the current methodology is already to a large extent robust with regards to the estimated parameters (Wuyts et al. 2003).

Traffic flows on a highway during non-congested hours are best described using a $M / G / 1$ queueing model in most cases. During the congested hours, the state dependent queueing $G I / G / z$ models are more realistic. Moreover, the Theil decomposition shows that the largest part of the errors made are random. Results showed that the queueing models developed can be adequately used to model uninterrupted traffic flows. As such, these models can be used to evaluate potential improvements in existing traffic conditions. Starting from the existing traffic state, potential improvements are easily quantified and compared with one another. Improvements that can be evaluated using this queueing approach are e.g. congestion pricing (Van Woensel et al. 2005), environmental impact of traffic (Van Woensel et al. 2001), optimal number of lanes, investment analysis, etc. Moreover, due to the analytical character of the queueing models developed, they are very suitable to be incorporated in other models, e.g., VRP models. 


\section{References}

Akçelik R (1991) Travel time functions for transport planning purposes Davidson's function, its timedependent form and an alternative travel time function. Australian Road Research 21: 49-59

Akçelik R (1996) Relating flow, density, speed and travel time models for uninterrupted and interrupted traffic. Traffic engineering and control 37: 511-516

Balci O (1998) Verification, validation and testing. In: Banks J (ed) Handbook of simulation: Principles, methodology, advances, applications and practice. Wiley, New York

Barceló J (2001) Dynamic network simulation with AIMSUN. Traffic Simulation Systems

Button KJ (1993) Transport economics. Edgar Elgar Publishing, Cambridge

Daganzo CF (1997) Fundamentals of transportation and traffic operations. Elsevier Science, Oxford, UK

Greenshields BD (1935) A study of traffic capacity. Highway Research Board Proceedings 14: 448-477

Davidson KB (1991) The theoretical basis of a flow-travel time relationship for use in transportation planning. Australian road research 8: 32-35

Heidemann D (1991) Queue length and waiting-time distributions at priority intersections. Transportation Research B 25: 163-174

Heidemann D (1991) Queue length and delay distributions at traffic signals. Transportation Research B 28: $377-389$

Heidemann D (1996) A queueing theory approach to speed-flow-density relationships. Proceedings of the 13th International Symposium on Transportation and Traffic Theory. Lyon, France

Heidemann D, Wegmann H (1996) Queueing at Unsignalized Intersections. Transportation Research B 31: 239-263

Holden K, Peel DA, Thompson JL (1990) Economic forecasting: An introduction. Cambridge University Press, Cambridge

Ichoua S, Gendreau M, Potvin J (2003) Vehicle dispatching with time-dependent travel times. European Journal of Operational Research 144: 379-396

Jain R, MacGregor Smith J (1997) Modeling vehicular traffic flow using $M / G / C / C$ state dependent queueing models. Transportation Science 31: 324-336

Kingman JFC (1964) The single server queue in heavy traffic. Proceedings of the Cambridge Philosophical Society 57: 902-904

Kleijnen JPC (1995) Theory and methodology: Verification and validation of simulation models. European Journal of Operational Research 82: 145-162

Kraemer W, Lagenbach-Belz M (1976) Approximate formulae for the delay in the queueing system $G I / G / 1$. Congressbook of the eight international teletraffic congress. Melbourne, 235-1/8

Transportation Research Board (1996) Traffic flow theory: A state-of-the-art report

Law AM, Kelton WD (2000) Simulation modeling and analysis. McGraw Hill, Singapore

Theil H (1966) Applied economic forecasting. North-Holland, Amsterdam

Vandaele N, Van Woensel T, Verbruggen A (2000) A queueing based traffic flow model. Transportation Research D 5: 121-135

Van Woensel T (2003) Models for uninterrupted traffic flows: a queueing approach. Phd Thesis, Department of Applied Economics, UFSIA, Antwerp, Belgium

Van Woensel T, Creten R, Vandaele N (2001) Managing the environmental externalities of traffic logistics: The issue of emissions. Production and Operations Management, Special issue on Environmental Management and Operations 10: 207-223

Van Woensel T, Wuyts B, Vandaele N (2005) A queueing approach to congestion pricing. Under review Whitt W (1993) Approximations for the $G I / G / m$ Queue. Production and Operations Management 2: 114-161

Wuyts B, Van Woensel T, Vandaele N (2003) State dependent queueing models for traffic. Research paper, University of Antwerp, Faculty of Applied Economics 